\section{Distilled delight}

\section{D.H. Perkins}

The Experimental Foundatlons of Particle

Physics. By Robert N. Cahn and Gerson Goldhaber. Cambridge University Press: 1989. Pp. 428. £30, $\$ 49.50$.

Progress in physical science depends on the close interplay between experiment and theory, and nowhere has this been more true than in the physics of elementary particles. Many standard texts on this subject tend to emphasize the beauty, conciseness and all-embracing nature of theoretical principles and ideas, so it is a pleasure to review a text in which, for a change, discussion of experiments takes pride of place.

Cahn and Goldhaber describe key experiments in twelve areas of particle physics, covering the discovery, over the years, of the various lepton and quark constituents of matter and the nature of the interactions between them. The authors have struck an excellent balance in what must have been a difficult choice of material.

The book is intended for advanced undergraduates and graduates in physics. The text in each chapter introduces a particular area of the field, presenting the basic physical concepts and background information necessary for an understanding of the significance of, typically, three or four original key papers, which are reprinted at the end of each chapter. The contents range from discovery of the neutron, positron, muon and pion in the 1930 s and 1940 s, to the study of "strange" particles and hadron resonances in the 1950 s and 1960s, which led to the quark model and was crowned with the discovery of the massive "charm" and "beauty" states in the 1970s. There are excellent chapters on the study of weak interactions, of the strong forces between quarks, and on the discovery of neutral currents and the $\mathrm{W}$ and $\mathrm{Z}$ bosons validating the electroweak model unifying the electromagnetic and weak interactions.

I believe the authors' assessment of the role of the various experiments to be well considered, authoritative and scrupulously fair. I have to say, however, that I sometimes found the balance between the space devoted to different topics to be slightly odd. The discovery at CERN of W and $\mathrm{Z}$ bosons - which were a crucial prediction of the electroweak model and for which the scientific world had waited 15 years - is covered in $1 \frac{1}{2}$ pages of text, whereas the Stanford discoveries of charm quarks and the tau-lepton - also very important - get 20 pages. Presumably this simply reflects the north Californian origin of the authors. There are few faux pas, although omitting the name of Bruno
Touschek in a discussion of colliders must be one of them.

Included at the end of each chapter is a set of problems. Many of these are well beyond undergraduate level and they are mostly of a theoretical nature. What a pity that, in a text devoted to the experimental foundations of the subject, there are not more practical exercises which involve calculating a real number! My only other criticism is that a little discussion of accelerator and detector technology and development would have been useful, as these form the backbone of the experimental scene. Regarding the quality of

presentation, I have to say that an otherwise well-produced book was for me marred by the poor quality of reproduction of the original papers, particularly in the first three chapters.

In summary, I believe this text to represent a major achievement in collecting, analysing and distilling for the reader, material forming an intensely exciting chapter in modern science.

D. H. Perkins is Professor of Elementary Particle Physics and head of the Nuclear Physics Department at the University of Oxford, Keble Road, Oxford OX1 3RH, UK.

\section{Spinning out}

\section{P.J. Hore}

One and Two Dimensional NMR Spectroscopy. By Atta-ur-Rahman. Elsevier: 1989. Pp.578. Dfl.355, \$186.75.

NUCLEAR magnetic resonance (NMR) spectroscopy has changed almost beyond recognition in the past decade. A glance through the literature reveals a truly staggering array of ingenious and specialized techniques for determining the structures and motions of molecules. Assisted by developments in computer and magnet technology, this metamorphosis has been made possible by the ease with which longlived coherent superpositions of nuclear spin states (coherences) can be created and manipulated using pulses of coherent, monochromatic, radiofrequency radiation.

No longer are chemists content simply to monitor the equilibrium state of the nuclear spins in a molecule subject to the restrictions of spectroscopic selection rules; rather they tailor the spectrum to suit their needs, shifting spin polarizations and coherences around the molecule so as to map out its chemical structure. A crucial factor in this revolution has been the move away from the conventional onedimensional approach. Two-dimensional NMR, in which signals are functions of two frequency variables, is now routine, while the first forays into a third frequency dimension have occurred in the past two years. The relief of crowding in the spectra of complex molecules afforded by twodimensional methods, together with their ability to reveal correlations between nuclei, have led to previously undreamt of achievements, most notably the determination of the complete three-dimensional structure of small proteins.

To take the greatest advantage of these new NMR techniques, the chemist needs to understand the physics underlying them. Atta-ur-Rahman's book is one of a handful of recent publications that aim to provide such insight, while also offering advice on the choice of experiment and guidance on interpretation of spectra. The book is restricted to proton and carbon-13 NMR of liquids, and deals with essentially every significant experiment up to about 1986. An undergraduate knowledge of NMR is assumed, but not much else. Most chapters finish with exercises in spectral interpretation chosen from the author's own research in natural product chemistry.

Atta-ur-Rahman succeeds in conveying the scope and power of one- and twodimensional NMR and shows clearly how structural information can be extracted. But he is less convincing when discussing how radiofrequency pulse sequences actually work. This he does by means of Felix Bloch's semi-classical vector model which, although simple and pictorial, cannot satisfactorily handle multiple quantum coherence or coherence transfer. As most modern techniques involve one or both of these elements, this is really a severe limitation. A newcomer to modern NMR would surely be better advised to embrace the product operator formalism to which the author devotes his final, short chapter. Once mastered, this approach requires only simple algebra and determination to enable the inner workings of complex experiments to be seen.

Sadly, there is a sufficient number of typographical errors to be irritating and, in places, confusing or misleading. Nor is the reader helped by a poorly organized introductory chapter and a rather imprecise style of writing. In short, this is a comprehensive and much-needed book, but it has serious flaws.

P. J. Hore is in the Physical Chemistry Laboratory, University of Oxford, South Parks Road, Oxford OX1 $3 Q Z$, UK.

- Two new volumes in the series Methods in Enzymology bear witness to the remarkable transformation in the practical application of NMR spectroscopy in the past 10 years. Volume 176, Nuclear Magnetic Resonance part A, covers basic techniques and methods for studying protein dynamics. Volume 177 (part B) describes protein modifications. Both volumes are published by Academic; part A is $\$ 65, £ 46.50$, part B (to be published in January 1990) is $\$ 65$, $£ 46.50$.

NATURE · VOL $342 \cdot 21 / 28$ DECEMBER 1989 